\title{
Single Cell Micro-Pillar-Based Characterization of Endothelial and Fibroblast Cell Mechanics
}

\author{
Julia Eckert ${ }^{1,+}\left(\mathbb{D}\right.$, Yasmine Abouleila ${ }^{2,+}\left(\mathbb{C}\right.$, Thomas Schmidt $^{1}\left(\mathbb{D}\right.$ and Alireza Mashaghi ${ }^{2, *}$ (i) \\ 1 Physics of Life Processes, Leiden Institute of Physics, Leiden University, 2333 CC Leiden, The Netherlands; \\ eckert@physics.leidenuniv.nl (J.E.); schmidt@physics.leidenuniv.nl (T.S.) \\ 2 Medical Systems Biophysics and Bioengineering, Leiden Academic Centre for Drug Research, \\ Leiden University, 2333 CC Leiden, The Netherlands; y.a.m.i.abouleila@lacdr.leidenuniv.nl \\ * Correspondence: a.mashaghi.tabari@lacdr.leidenuniv.nl \\ + These authors contributed equally to this work.
}

Citation: Eckert, J.; Abouleila, Y.;

Schmidt, T.; Mashaghi, A. Single Cell Micro-Pillar-Based Characterization of Endothelial and Fibroblast Cell Mechanics. Micro 2021, 1, 242-249. https://doi.org/10.3390/micro1020 018

Academic Editor: Ilaria Tonazzini

Received: 20 October 2021

Accepted: 20 November 2021

Published: 25 November 2021

Publisher's Note: MDPI stays neutral with regard to jurisdictional claims in published maps and institutional affiliations.

Copyright: (C) 2021 by the authors. Licensee MDPI, Basel, Switzerland. This article is an open access article distributed under the terms and conditions of the Creative Commons Attribution (CC BY) license (https:/ / creativecommons.org/licenses/by/ $4.0 /)$.

\begin{abstract}
Mechanotransduction, the ability of cells to sense and respond to the mechanical cues from their microenvironment, plays an important role in numerous cellular processes, ranging from cell migration to differentiation. Several techniques have been developed to investigate the underlying mechanisms of mechanotransduction, in particular, force measurement-based techniques. However, we still lack basic single cell quantitative comparison on the mechanical properties of commonly used cell types, such as endothelial and fibroblast cells. Such information is critical to provide a precedent for studying complex tissues and organs that consist of various cell types. In this short communication, we report on the mechanical characterization of the commonly used endothelial and fibroblast cells at the single cell level. Using a micropillar-based assay, we measured the traction force profiles of these cells. Our study showcases differences between the two cell types in their traction force distribution and morphology. The results reported can be used as a reference and to lay the groundwork for future analysis of numerous disease models involving these cells.
\end{abstract}

Keywords: traction force; cell mechanics; single-cell analysis; endothelial cell; fibroblast

\section{Introduction}

Mechanics is a fundamental property of biological cells with implications for various biological functions, ranging from single cell migration to organ-level functions, such as tissue barrier integrity regulation. The emergence of bio-printed and organ on chip models was a response to the need for modeling of mechanical alterations in numerous diseases [1]. In particular, mechanical dysregulation of endothelial cells is involved in several functions and is attributed to various conditions including, autoimmune vasculopathies, viral hemorrhagic syndromes, allergic reactions, and cancer. Similar to endothelial cells, mechanical regulation of fibroblasts is involved in various cellular functions. Among these are, extracellular matrix (ECM) remodeling [2], tissue regeneration [3] and angiogenesis [4]. Mechanotransduction has been closely linked to inflammation, wound healing and fibrosis $[5,6]$. There is increasing evidence that mechanical forces heavily influence all phases of wound healing, ranging from post-injury inflammation to fibrosis [7]. Mechanical cues from the microenvironment modulate vascular endothelium permeability and ECM synthesis. Stresses from the microenvironment are then translated to adhesion forces created between cells, and cellular traction forces applied on the ECM [8,9]. Traction forces generated by the actomyosin machinery contribute to the cellular mechanical properties, and they are believed to play pivotal roles in regulating various cellular mechanosensing processes, such as cell differentiation, migration and proliferation.

Several approaches attempted to characterize the cellular traction forces. One pronounced methodology is micropillar array substrates. Through selective coating of the tips of the pillars with ECM proteins, cells are allowed to adhere and exert forces on 
them, which results in pillar deflections that correspond to intracellular traction forces. Here, we build upon our recent work and developed an in vitro assay to quantitatively compare the mechanical properties of two cell types, endothelial cells and fibroblasts at the single cell scale. We characterized two commonly used cell models, the human umbilical vein endothelial cells (HUVECs) and 3T3 fibroblast cells. Both are established models for studying fibroblast and vascular biology in health and disease [10]. Our results showcase discrepancies in the distribution of traction forces among the two cell types. Endothelial cells appeared to exert lower traction forces on the ECM substrate when compared to fibroblast cells. Additionally, differences in cellular morphology were observed, where a lower cell-eccentricity was detected in endothelial cells in comparison to fibroblast cells. Both cell types exert dipolar forces, however, an additional three-fold symmetry was identified for fibroblast cells in certain cell-eccentricity ranges.

\section{Materials and Methods}

\subsection{Cell Culture}

3T3 fibroblast cells were cultured in high-glucose Dulbecco Modified Eagle's Medium (D6546; Sigma-Aldrich, St. Louis, MO, USA) supplemented with 10\% fetal calf serum (Thermo Fisher Scientific, Waltham, MA, USA), $2 \mathrm{mM}$ glutamine, and $100 \mathrm{mg} / \mathrm{mL}$ penicillin/streptomycin, $37^{\circ} \mathrm{C}, 5 \% \mathrm{CO}_{2}$. For HUVECs, cells were cultured in Endothelial Cell Basal Medium 2 (PromoCell, C-22211) and supplemented with Growth Medium 2 SupplementMix (PromoCell, C-39216) and $100 \mathrm{mg} / \mathrm{mL}$ penicillin/streptomycin.

\subsection{Immunostaining}

After $22.5 \mathrm{~h}$ of spreading, 3T3 fibroblast and HUVEC cells were fixed for $15 \mathrm{~min}$ in 4\% paraformaldehyde (43368; Alfa Aesar, Haverhill, MA, USA) in phosphate-buffered saline (PBS). Furthermore, cells were permeabilized for $10 \mathrm{~min}$ with $0.1 \%$ Triton-X 100 in $1 \%$ bovine serum albumin (BSA) and blocked for 60 min with 1\% BSA in PBS. F-actin was stained with Alexa Fluor 532-labeled phalloidin (A22282; Invitrogen, Carlsbad, CA, USA) and the DNA with DAPI (Sigma-Aldrich).

\subsection{Elastic Micropillar Arrays}

Polydimethylsiloxane (PDMS, Sylgard 184) micropillar arrays of $2 \mu \mathrm{m}$ diameter, $6.9 \mu \mathrm{m}$ length, and $4 \mu \mathrm{m}$ spacing in a hexagonal geometry were used for cell traction force experiments. The pillar arrays were flanked by $50 \mu \mathrm{m}$ spacers on two sides of the array. Details of this arrangement and the experimental procedures were described earlier in detail [11]. In brief, pillar arrays were produced on a negative silicon-wafer master made by a two-step deep reactive-ion etching process. Wafers were passivated in trichloro-silane (448931; Sigma-Aldrich). A mixture of 1:10 PDMS (cross-linker/base ratio) was poured onto the Si-master and cured for $20 \mathrm{~h}$ at $110^{\circ} \mathrm{C}$. After peeling off, the tops of the pillars were coated by micro-contact printing. For that, flat 1:30 PDMS stamps were incubated for $1 \mathrm{~h}$ with $40 \mathrm{~mL}$ of $50 \mathrm{mg} / \mathrm{mL}$ Alexa Fluor $647-$ labeled and $50 \mathrm{mg} / \mathrm{mL}$ unlabeled fibronectin (F1141; Sigma-Aldrich), then washed and dried. Subsequently, the stamps were gently loaded onto the ultraviolet-ozone-activated micropillar arrays for $10 \mathrm{~min}$. After stamping, the arrays were passivated with $0.2 \%$ Pluronic (F-127, P2443; Sigma-Aldrich) for $1 \mathrm{~h}$, and washed in PBS.

\subsection{Microscopy}

Samples were imaged at high resolution on a home-build optical microscope setup based on an inverted Axiovert200 microscope body (Carl Zeiss, Oberkochen, Germany), a spinning disk unit (CSU-X1; Yokogawa Electric, Musashino, Tokyo, Japan), and an emCCD camera (iXon 897; Andor Labs, Morrisville, NC, USA). IQ-software (Andor Labs) was used for setup-control and data acquisition. Illumination was performed using fiber-coupling of different lasers (405 nm (CrystaLaser, Reno, NV, USA), $514 \mathrm{~nm}$ (Cobolt AB, Solna, Sweden), and $642 \mathrm{~nm}$ (Spectra-Physics Excelsior; Spectra-Physics, Stahnsdorf, Germany)). Pillar 
arrays were placed upside down onto $25 \mathrm{~mm}$ cover glasses and inspected with an EC Plan-NEOFLUAR 40 1.3 Oil Immersion Objective (Carl Zeiss).

\subsection{Image Analysis}

Images of single, nonoverlapping and randomly selected cells within the field of view of $176 \times 176 \mu \mathrm{m}$ were analyzed using MATLAB scripts (MATLAB R2018a; MathWorks, Natick, MA, USA). Pillar deflections were quantified as previously described in detail [11]. Deflected pillars caused by cell traction forces were distinguished from the background. The background was determined from an undeflected area of the pillar array by selecting a pillar region outside the cell area. Pillar deflections underneath the cell within the background range were excluded.

The cell spreading morphology was characterized by the moment of inertia of an ellipse using the Regionprops function in MATLAB. In respect to the minor axis of the ellipse, we measured the angular position $\left(0^{\circ}\right.$ to $\left.360^{\circ}\right)$ of the deflected pillars around the nucleus. The angular position $0^{\circ}$ was chosen in such a way that most deflected pillars were close to the major axis at $270^{\circ}$ and less at $90^{\circ}$ (Figure 2D).

\subsection{Statistics}

In total, we analyzed 133 HUVEC cells from eight samples of four independent performed experiments and $3233 \mathrm{~T} 3$ fibroblasts from six independent samples.

All data sets are of non-normal distribution. The $p$-values were calculated using the two-sided Wilcoxon rank sum test in MATLAB. Data sets were significantly different with probabilities of $p<0.0001\left(^{* * * *)} ; p>0.05(\mathrm{~ns})\right.$.

\section{Results}

\subsection{Endothelial Cells Apply Less Traction Forces Compared to Fibroblast Cells}

Previously, we have shown that the total traction force of single 3T3 fibroblast cells exerted on fibronectin-coated micropillars is proportional to the number of deflected pillars [12]. To validate this behavior for endothelial cells, we measured the traction force of 133 HUVEC cells (Figure 1A). The force correlated highly to the number of deflected pillars per cell with a correlation coefficient of $r=0.9$ (Figure 1B). The linear dependence between the number of deflected pillars and the total traction force results in a single parameter for cellular traction force characterizations, the mean traction force per deflected pillar. For HUVEC cells, we measured a mean traction force per pillar of $6.9 \pm 1.9 \mathrm{nN}$ (mean \pm s.d.) (Figure 1D). This value is significantly smaller than the averaged force per pillar of $12.6 \pm 5.2 \mathrm{nN}$ (mean \pm s.d.) applied by $3 \mathrm{~T} 3$ fibroblasts $(p<0.0001)$. We should note, that the number of deflected pillars for the HUVEC cells correlated with the total number of pillars per cell $(r=0.7)$, i.e., the cell spreading area (Figure 1C), a result that we reported also for fibroblasts earlier [12]. The averaged cell spreading area was significantly higher for HUVEC cells with $3542 \pm 1486 \mu \mathrm{m}^{2}$ (mean \pm s.d.) compared to $3 \mathrm{~T} 3$ fibroblasts with $1328 \pm 673 \mu \mathrm{m}^{2}$ (mean \pm s.d.) (Figure 1E).

\subsection{Averaged Force Dipole Distribution Is Independent of Cell Type}

It is known that non-rounded cells generate force dipoles due to the contractility of their actomyosin machinery $[13,14]$. In round-shaped cells, forces are uniformly applied on substrates and mainly distributed at the cell-periphery [15]. We measured the positions of deflected pillars according to the cell morphology. The cell spreading morphology was characterized by the moments-of-inertia of the cell shape approximated by an ellipse (Figure 2A endothelial cells, Figure 2D fibroblasts). With respect to the minor axis of the ellipse, we measured the angular position of the deflected pillars around the nucleus (Figure 2B endothelial cells, 15,682 deflections, Figure 2E fibroblasts, 8824 deflections). Both distribution of the angular positions for 133 analyzed endothelial cells and 323 fibroblasts show two peaks at an angular distance of $180^{\circ}$, hence located at the ends of the major axes. Together with the high eccentricities of both cell lines (Figure 2C endothelial cells, 
Figure 2F fibroblasts), the data show that both, endothelial cells and fibroblast cells, generally form force-dipoles. It should be noted that the distributions around the main peaks in endothelial cells are broader compared to those of fibroblasts, indicating a difference in the cell spreading morphology. Endothelial cells are less elongated and tend to be rounder (Figure 2C). In contrast, fibroblast cells are more elongated (Figure 2F) and form narrow force dipoles (Figure 2E), which can be seen from the high probability distribution for larger eccentricities. Furthermore, we did not observe significant difference in circularity between 3 T3 fibroblasts with $0.17 \pm 0.06$ (mean \pm s.d.) and HUVEC cells with $0.17 \pm 0.08$ (mean \pm s.d.) (Figure 1F).

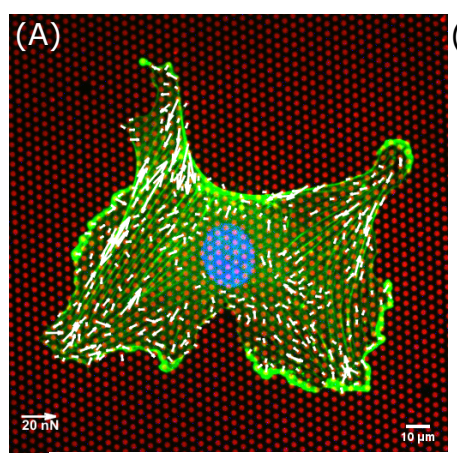

(D)

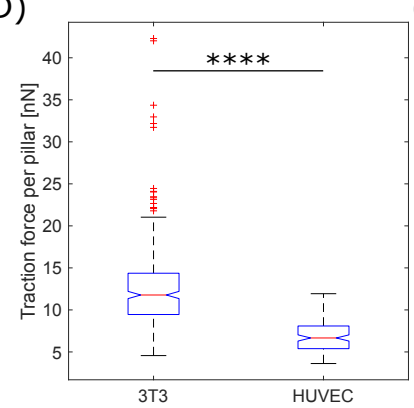

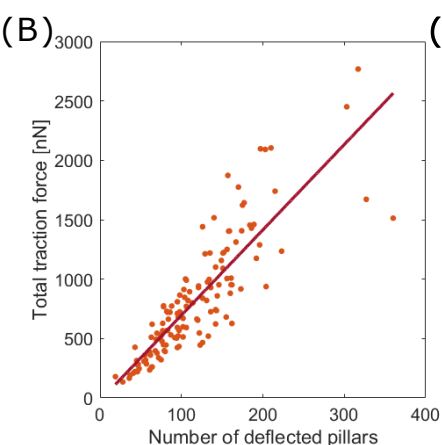

(E)

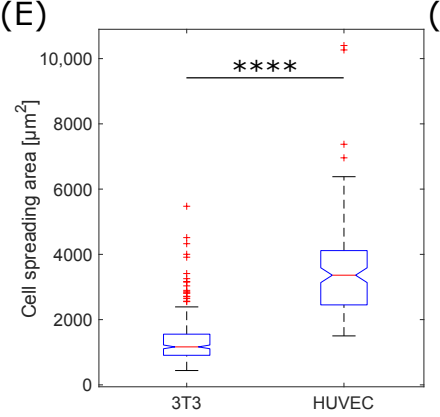

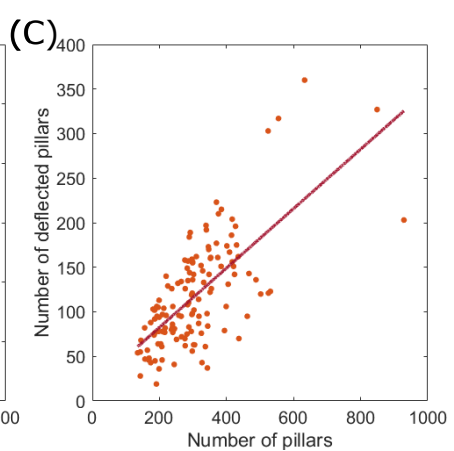

(F)

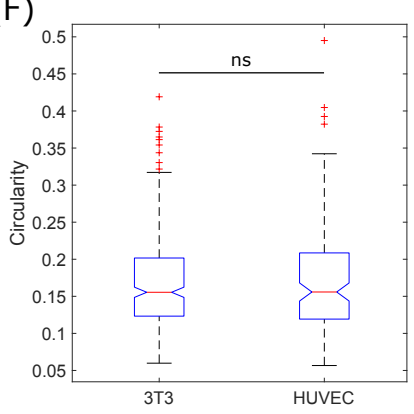

Figure 1. Traction forces are significantly higher for fibroblasts compared to endothelial cells. (A) A single endothelial cell on fibronectin-coated micropillars (red) showing F-actin (green), the nucleus (blue) and traction forces (white). (B) Total traction force per endothelial cell as a function of the number of deflected pillars. (C) Number of deflected pillars per endothelial cell correlated with the number of pillars per cell. (D) Mean traction force per deflected pillar per cell for fibroblasts and endothelial cells. (E) Cell spreading area. (F) Circularity. ns = not significant; ${ }^{* * * *} p<0.0001$ using the two-sided Wilcoxon rank sum test.

\subsection{Force Pole Is Cell Morphology and Cell Type Dependent}

As a next step, we investigated the dependence of the force distribution on the cell morphology in more detail. We compared the polarity of endothelial cells and fibroblast cells with their eccentricity for similarities and differences (Figure 3A-C endothelial, Figure 3D-F fibroblast). First, we investigated whether we could subtract the triangular shape of fibroblast cells from our data [16]. We plotted the angular distribution for different eccentricities, $\epsilon$, and identified three peaks at an eccentricity range between 0.8 and 0.9 (Figure 3E, orange arrows, 54 cells, 1650 deflections), indicating a three-pole force distribution. In comparison, highly elongated cells, $0.9<\epsilon \leq 1$, exerted mainly traction forces on their major axes, forming sharp force dipoles (Figure 3F, 211 cells, 5691 deflections). Endothelial cells, in contrast, retained their dipole distribution in all eccentricity-ranges, even when the distribution became more uniform for rounder cells $(0.8<\epsilon \leq 0.9$ : 40 cells, 5436 deflections; $0.9<\epsilon \leq 1$ : 37 cells, 3888 deflections. 

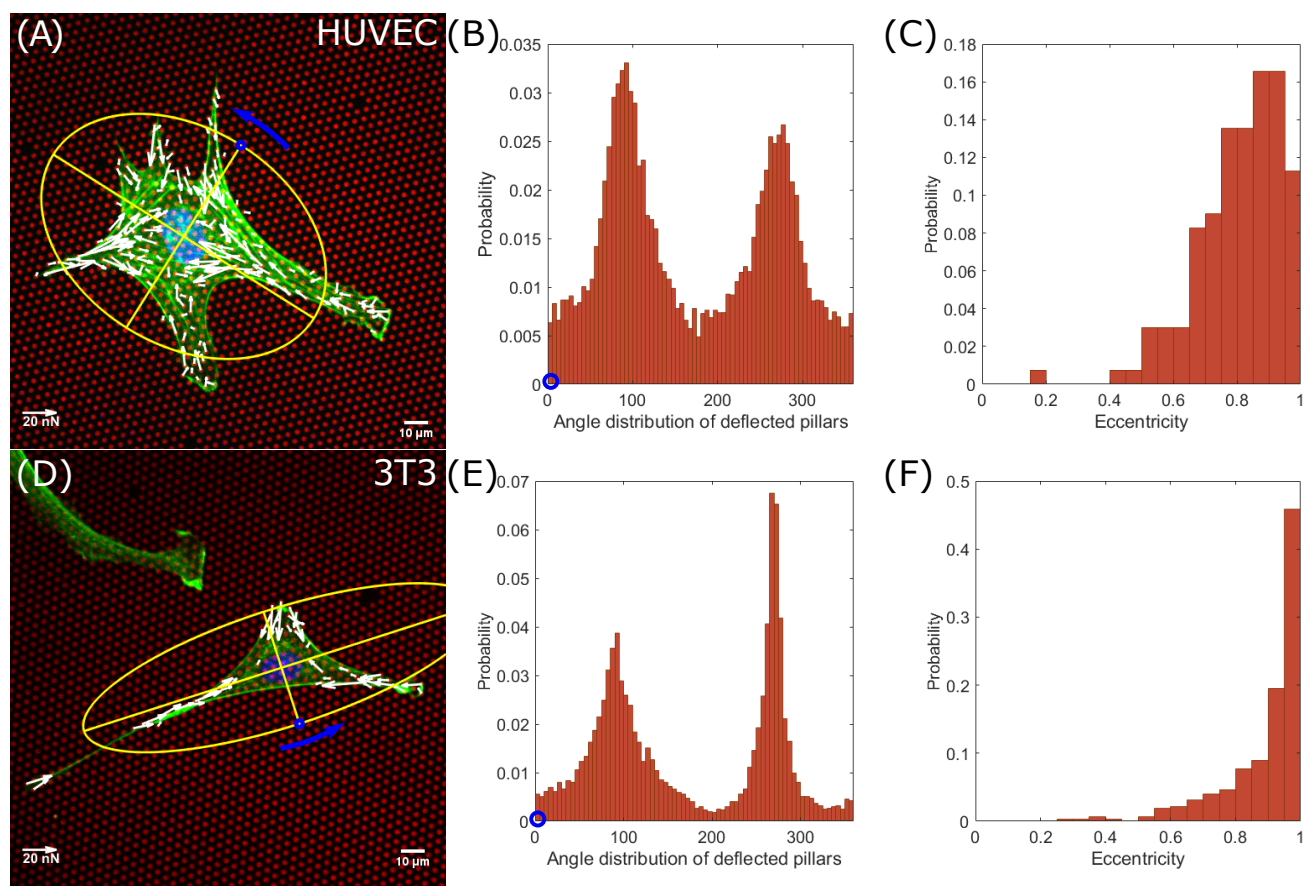

Figure 2. Endothelial cells and fibroblasts have dipolar force distributions. Angular distribution of deflected pillars and morphology analysis of endothelial cells (A-C) and fibroblast cells (D-F). (A,D) Single cells on fibronectin-coated micropillars (red) showing F-actin (green), the nucleus (blue) and traction forces (white). The cell spreading morphology was characterized by the moment of inertia of an ellipse (yellow). (B,E) Distributions of deflected pillars were assigned by counterclockwise rotation around the nucleus, starting at the short axis (blue circle). (C,F) Probability distributions of eccentricities.

(A)

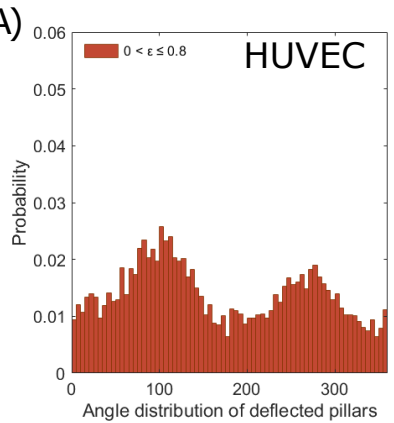

(D)

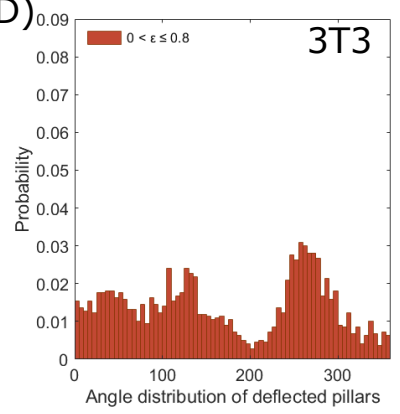

(B)

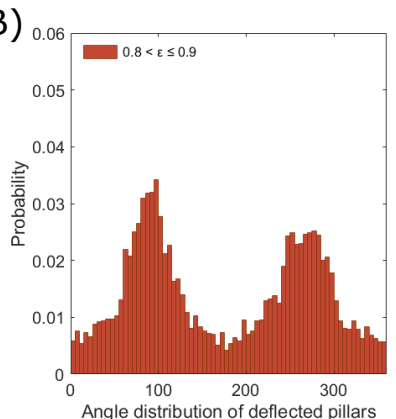

(E)

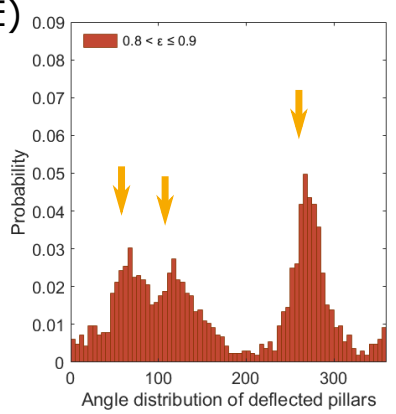

(C)

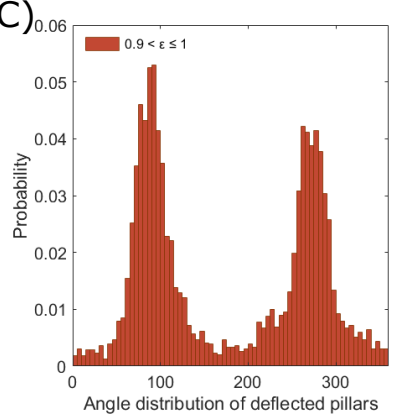

$(F)_{0}$

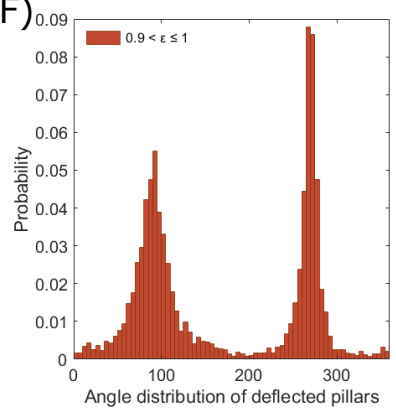

Figure 3. The force distribution pattern depends on the eccentricity of cells. Angular distribution of deflected pillars according to different eccentricities, $\epsilon$, of endothelial cells (A-C) and fibroblast cells (D-F). Endothelial cells retain two distribution peaks for all shown eccentricity ranges. Fibroblast cells show three deflection peaks (yellow arrows) at $0.8<\epsilon \leq 0.9$ (E) and two peaks at $0.9<\epsilon \leq 1$ (F). 


\section{Discussion}

Cellular mechanics is far from being homogenous across different cells. Mechanical heterogeneities of cells have been reported to largely influence several cellular processes, including response and resistance to treatment, mechanotransduction and tumor metastasis [17]. As such, studying the mechanical properties of individual cells is a prerequisite to provide relevant insights into the prevention and treatment of disease. Here, using a micropillar-based assay, we quantitively describe the traction forces exerted by single endothelial cells and compared those to fibroblast cells. We identified particular differences between both cell types according to their traction force distribution and morphology. Endothelial cells were found to exert overall lower traction forces on the ECM substrate (mean traction force of $6.9 \pm 1.9 \mathrm{nN}$ ) when compared to fibroblasts $(12.6 \pm 5.2 \mathrm{nN})$. This may result from endothelial cells seeking cell-cell connections necessary for network formation, and hence resulting in stronger cell-cell adhesion forces, rather than cell-substrate adhesion. In addition, it was reported earlier that traction forces scale with focal adhesion areas [18-22]. Therefore, compared to fibroblasts, endothelial cells might create less focal adhesions, which means that less traction forces are applied on substrates. Despite this, endothelial network formation relies on a balance between cell-cell and cell-substrate force interactions [23]. While, in the case of fibroblast cells, stronger cell-substrate interactions are expected, given that fibroblasts are known to be largely responsible for ECM synthesis and remodeling [24]. We further identified a significant heterogeneity in the mean force per pillar in both endothelial and fibroblast cells. The heterogeneity in cell binding to the ECM as dictated by specific cell-ECM interactions will contribute to specific ECM remodeling, that is subsequently resulting in the structural heterogeneity of the ECM [25].

Regarding to the cellular morphology, individual endothelial cells appeared to be more circular in their morphology when compared to fibroblasts. These morphological differences, quantified here as the cells' eccentricity, were found to be reflected in the angular force distribution as well. A broader force distribution pattern was detected in case of endothelial cells when compared to fibroblasts, indicating a difference in cell spreading morphology. Both cells exhibited a dipolar force distribution, which corroborates previous studies, that migrating cells (e.g., endothelial cells and fibroblasts) demonstrate a dipolar behavior [26]. In addition, we observed a tri-polar force distribution specific to fibroblasts at a narrow eccentricity range ( 0.8 to 0.9$)$. We speculate that the correlation of the force distribution-pattern with cellular morphology could lead to guide the directionality of cell movements, an insight that may be important for the mechanism of cell migration.

In conclusion, the micropillar-based assay reported here provides a reference for singlecell mechanical data for two commonly used cell types, HUVEC cells and 3T3 fibroblasts. We believe that our results will be of interest for future studies on the mechanics of complex tissues and organs involving these cell models. Recent studies showed a possible link between endothelial cells and fibroblasts functions in events like, inflammation and wound healing $[27,28]$. Thus, this assay and our data can be extended further to include cellcell interactions to help understand how endothelial cells and fibroblasts interact and coordinate their dynamics during said events.

Author Contributions: Cell culture and sample preparation of 3T3 cells, J.E.; microfabrication of micropillar arrays, data analysis, J.E.; cell culture and sample preparation of HUVECs, Y.A.; manuscript writing, J.E., Y.A. and A.M.; supervising the project, T.S. and A.M. All authors have read and agreed to the published version of the manuscript.

Funding: This research was funded by Leiden University Fund grant number W19340-5-EML and the Netherlands Organisation for Scientific Research grant numbers NWA.1228.191.329 and OCENW.XS4.180.

Institutional Review Board Statement: The study was conducted according to the guidelines of the Declaration of Helsinki.

Informed Consent Statement: Not applicable. 
Data Availability Statement: The original data are available on reasonable request to the corresponding author.

Conflicts of Interest: The authors declare no conflict of interest. The funders had no role in the design of the study; in the collection, analyses, or interpretation of data; in the writing of the manuscript, or in the decision to publish the results.

\section{References}

1. Evers, T.M.J.; Holt, L.J.; Alberti, S.; Mashaghi, A. Reciprocal regulation of cellular mechanics and metabolism. Nat. Metab. 2021, 3, 456-468. [CrossRef]

2. Leslie, M.N.; Chou, J.; Young, P.M.; Traini, D.; Bradbury, P.; Ong, H.X. How Do Mechanics Guide Fibroblast Activity? Complex Disruptions during Emphysema Shape Cellular Responses and Limit Research. Bioengineering 2021, 8, 110. [CrossRef]

3. Li, B.; Wang, J.H.C. Fibroblasts and myofibroblasts in wound healing: force generation and measurement. J. Tissue Viability 2011, 20, 108-120. [CrossRef] [PubMed]

4. Sorrell, J.M.; Baber, M.A.; Caplan, A.I. A Self-Assembled Fibroblast-Endothelial Cell Co-Culture System That Supports in vitro Vasculogenesis by both Human Umbilical Vein Endothelial Cells and Human Dermal Microvascular Endothelial Cells. Cells Tissues Organs 2007, 186, 157-168. [CrossRef] [PubMed]

5. Chaudhuri, O.; Cooper-White, J.; Janmey, P.A.; Mooney, D.J.; Shenoy, V.B. Effects of extracellular matrix viscoelasticity on cellular behaviour. Nature 2020, 584, 535-546. [CrossRef] [PubMed]

6. Henderson, N.C.; Rieder, F.; Wynn, T.A. Fibrosis: From mechanisms to medicines. Nature 2020, 587, 555-566. [CrossRef]

7. Kuehlmann, B.; Bonham, C.A.; Zucal, I.; Prantl, L.; Gurtner, G.C. Mechanotransduction in Wound Healing and Fibrosis. J. Clin. Med. 2020, 9, 1423. [CrossRef]

8. Arslan, F.N.; Eckert, J.; Schmidt, T.; Heisenberg, C.P. Holding it together: When cadherin meets cadherin. Biophys. J. 2021, 120, 4182-4192. doi: 10.1016/j.bpj.2021.03.025. [CrossRef] [PubMed]

9. Lemmon, C.A.; Sniadecki, N.J.; Ruiz, S.A.; Tan, J.L.; Romer, L.H.; Chen, C.S. Shear force at the cell-matrix interface: enhanced analysis for microfabricated post array detectors. Mech. Chem. Biosyst. MCB 2005, 2, 1-16.

10. Medina-Leyte, D.J.; Domínguez-Pérez, M.; Mercado, I.; Villarreal-Molina, M.T.; Jacobo-Albavera, L. Use of Human Umbilical Vein Endothelial Cells (HUVEC) as a Model to Study Cardiovascular Disease: A Review. Appl. Sci. 2020, 10, 938. [CrossRef]

11. van Hoorn, H.; Harkes, R.; Spiesz, E.M.; Storm, C.; van Noort, D.; Ladoux, B.; Schmidt, T. The Nanoscale Architecture of Force-Bearing Focal Adhesions. Nano Lett. 2014, 14, 4257-4262. [CrossRef]

12. Eckert, J.; van Loon, J.J.A.; Eng, L.M.; Schmidt, T. Hypergravity affects cell traction forces of fibroblasts. Biophys. J. 2021, 120, 773-780. doi: 10.1016/j.bpj.2021.01.021. [CrossRef]

13. Dembo, M.; Wang, Y.L. Stresses at the cell-to-substrate interface during locomotion of fibroblasts. Biophys. J. 1999, 76, 2307-2316. [CrossRef]

14. Schwarz, U.S.; Safran, S.A. Elastic Interactions of Cells. Phys. Rev. Lett. 2002, 88, 048102. [CrossRef]

15. Abuhattum, S.; Gefen, A.; Weihs, D. Ratio of total traction force to projected cell area is preserved in differentiating adipocytes. Integr. Biol. Quant. Biosci. Nano Macro 2015, 7, 1212-1217. [CrossRef]

16. Abercrombie, M. Fibroblasts. J. Clin. Pathol. Suppl. R. Coll. Pathol. 1978, 12, 1-6.

17. Li, X.; Das, A.; Bi, D. Mechanical Heterogeneity in Tissues Promotes Rigidity and Controls Cellular Invasion. Phys. Rev. Lett. 2019, 123, 058101. [CrossRef] [PubMed]

18. Balaban, N.Q.; Schwarz, U.S.; Riveline, D.; Goichberg, P.; Tzur, G.; Sabanay, I.; Mahalu, D.; Safran, S.; Bershadsky, A.; Addadi, L.; et al. Force and focal adhesion assembly: a close relationship studied using elastic micropatterned substrates. Nat. Cell Biol. 2001, 3, 466-472. [CrossRef] [PubMed]

19. Tan, J.L.; Tien, J.; Pirone, D.M.; Gray, D.S.; Bhadriraju, K.; Chen, C.S. Cells lying on a bed of microneedles: An approach to isolate mechanical force. Proc. Natl. Acad. Sci. USA 2003, 100, 1484-1489. [CrossRef]

20. Shemesh, T.; Geiger, B.; Bershadsky, A.D.; Kozlov, M.M. Focal adhesions as mechanosensors: A physical mechanism. Proc. Natl. Acad. Sci. USA 2005, 102, 12383-12388. [CrossRef] [PubMed]

21. Trichet, L.; Le Digabel, J.; Hawkins, R.J.; Vedula, S.R.K.; Gupta, M.; Ribrault, C.; Hersen, P.; Voituriez, R.; Ladoux, B. Evidence of a large-scale mechanosensing mechanism for cellular adaptation to substrate stiffness. Proc. Natl. Acad. Sci. USA 2012, 109, 6933-6938. [CrossRef]

22. Balcioglu, H.E.; van Hoorn, H.; Donato, D.M.; Schmidt, T.; Danen, E.H.J. The integrin expression profile modulates orientation and dynamics of force transmission at cell-matrix adhesions. J. Cell Sci. 2015, 128, 1316-1326. [CrossRef] [PubMed]

23. Califano, J.P.; Reinhart-King, C.A. Exogenous and endogenous force regulation of endothelial cell behavior. J. Biomech. 2010, 43 1, 79-86. [CrossRef]

24. Rhee, S.; Grinnell, F. Fibroblast mechanics in 3D collagen matrices. Adv. Drug Deliv. Rev. 2007, 59, 1299-1305. [CrossRef] [PubMed]

25. Malandrino, A.; Mak, M.; Kamm, R.D.; Moeendarbary, E. Complex mechanics of the heterogeneous extracellular matrix in cancer. Extrem. Mech. Lett. 2018, 21, 25-34. doi: 10.1016/j.eml.2018.02.003. [CrossRef]

26. Mandal, K.; Wang, I.; Vitiello, E.; Orellana, L.A.C.; Balland, M. Cell dipole behaviour revealed by ECM sub-cellular geometry. Nat. Commun. 2014, 5, 5749. [CrossRef] [PubMed] 
27. Slany, A.; Bileck, A.; Kreutz, D.; Mayer, R.L.; Muqaku, B.; Gerner, C. Contribution of Human Fibroblasts and Endothelial Cells to the Hallmarks of Inflammation as Determined by Proteome Profiling. Mol. Cell. Proteom. 2016, 15, 1982-1997. [CrossRef]

28. Tefft, J.B.; Chen, C.S.; Eyckmans, J. Reconstituting the dynamics of endothelial cells and fibroblasts in wound closure. APL Bioeng. 2021, 5, 016102. [CrossRef] 